\title{
Predicting Market Response to Monetary Policy in Economic Crisis Phase and Deriving a Decision Support System with Artificial Neural Network
}

\author{
Vyom Shrivastava
}

\begin{abstract}
Economic bubbles are the inevitable part of our Economic system and are responsible for various past turbulence, which had a deep impact for several years. It is a very well established fact that the prediction of the economic bubbles is an extremely tough job because of the involvement of a lot of non-linear factors, therefore it could be extremely useful to have some kind of a decision support system which could throw some light on the market movements. Another challenge that $I$ intend to solve through this paper is pre-determining the response of the market to various variables, directly or indirectly controlled by the federal reserve bank and the central government. This paper attempts to present a clubbed variable which could be used in assessing the response of the market to the prevailing monetary policy. The research involves developing of an Artificial Neural Network Model to access the monetary policy response to the market and to create an ANN decision support mechanism for assisting traders, academicians and even the central bank for pre-determining the potential impact of the given monetary policy based on the historic data.

These models will not only reduce the uncertainty but the ANN algorithm will also capture complex trends and relationships which are impossible for an unaided trader to extract.
\end{abstract}

Index Terms-Artificial neural network, decision support system, economic bubbles, monetary policy

\section{INTRODUCTION}

Artificial Neural Networks or ANN are one of the most widely used Machine Learning Algorithms. It draws an analogy from human brain and the functioning of the neurons, which are basically the smallest unit of our brain. ANNs start by assigning random weights to the smallest units of the algorithm-Neurons. The ANN model, then starts learning and assign optimized weights. It is used in a spectrum of applications varying from speech recognition to genetics. Therefore, it is natural for such a powerful tool to enter the fascinating financial world. Financial world has always been very non linear. Given the dynamic nature of the financial time series, it is important to select a model which can deal with the non linear systems. Artificial Neural Network is one such model. It is a well known fact that ANN approach is

Manuscript received February 16, 2016; revised June 7, 2017.

Vyom Shrivastava is with department of Mechanical Engineering, IIT Kharagpur, India (e-mail: vyomiitkgp@gmail.com). very effective in time series modeling. This paper examines the clustering ability of the model with financial time series as input and output. The waviness of the financial number series is carefully fed into a designed Artificial Neural Network system (as described in the later sections) as a classification problem. ANN comes with a lot lesser number of parameters to tune as compared to other standard machine learning algorithms, which makes it the most appropriate model for a widespread use among traders, academicians and banks.

Our Economic system has witnessed several financial crises and economic bubbles and it is our deepest concern to improve our methodologies to tackle such future economic crashes. Many economists believe that the great crisis of 2008 was initiated because of the abrupt decrement in the fed rates from $6.5 \%$ to $1.75 \%$ in December 2001. The paper attempts to devise a whistle blower system which can capture such sudden and explicit movements which can cause a downgrading impact on the market.

According to the Keynesian approach, government stabilization activities and economic intervention can create a significant cushion to such economic crises. Robert Shiller, Yale Economist, argues that an economic crisis is often a result of Irrational Exuberance, Strong psychological pressures, Herding, Short-Termism, Adaptive expectations and, cognitive dissonance [1]. Most of the above-mentioned factors (directly or indirectly) are the result of the monetary policies which are regulated by Federal Open Market Community (FOMC).

Monetary policy is a set of actions taken by the central bank to keep running the economy in a prosperous manner. These 'set of actions' consists of modifying interest rates, buying and selling government bonds and regulating amounts of mandatory bank reserves.

Against the pessimistic approaches of the "Efficient Market Theory" [2] and the "Random Walk Theory" [3], I tried to develop an ANN model which will capture complex and non-linear market variations caused by the monetary policies in a crashing economic scenario. The results are in unison with Keynesian perspective.

With the current immense development in the ML (Machine Learning), it gives traders an enormous opportunity to leverage the technology to derive essentials insights into the undetermined irregularities of the market, especially in the crisis period.

This paper attempts to apply ANN [4], [5] for predicting the response of the market from the set monetary policy in an economic crash.

An Artificial Neural Network Decision support system is 
developed to predict the behavior of the market on the daily basis and it will explicitly categorize the overall monetary situation into SELL, BUY or HOLD trading day with an excellent decision accuracy of around $60 \%$ in a crisis.

\section{FEATURE SELECTION}

The data features are selected in such a manner that they capture maximum information of the prevailing monetary policy. As explained above, government regulates the economy by modifying federal interest rates, buying and selling government bonds and by varying the mandatory amount of bank reserves. Considering the dynamics of the Federal Bank in mind, Federal reserve rate, Banks Reserve Requirements and Federal Debt are chosen as features. To capture the implicit dynamics, Fed Financial Stress Index, Euro to Dollar foreign exchange rate, Consumer Price Index (CPI) and Gross Domestic Product (GDP) are also considered. S\&P 500 Index is used as the predictor of the market in our analysis.

All the data was gathered from the official website- FRED, Economic Research by Federal Reserve Bank of St. Louis, which a very popular data source for economic researchers and traders.

\section{DATA PREPROCESSING}

The data available in the FRED for the above mentioned seven features is not uniform. For some of the features, daily data is not available. As ANN is trained to think like a human brain, the algorithm is fed with the previous available value and therefore making all series on a daily basis except non trading days. A code was written in R-Studios to smoothen all the variables available and the voids were filled by the numbers of the prevailing data point.

\section{FED INDEX}

\section{A. Modeling}

Among our features- Federal Debt, Bank Reserve Requirements, Federal Reserve Rates, Fed Financial Stress Index, Exchange Rate, CPI and GDP, a good correlation of Federal Debt with GDP $(\sim 70 \%)$, Reserve Requirements ( $97 \%)$, Interest Rate $(\sim-88 \%)$ and, CPI $(83 \%)$ is observed. Some other variables were also seemed to be correlated and therefore, a new index, capturing the variance and trends of all the mentioned variables. We name it FED INDEX, as it directly depicts the state of the prevailing monetary policy.

Principal Component Analysis, as stated by Svante Wold (1987) is an efficient way to scale down multi-dimensional problem for dimensionality reduction. Principal Component Analysis (PCA) was done on our Master Dataset and results were very revealing. The dimensionality of the whole federal index could be reduced to one dimension (First principal Component) without losing more than $2 \%$ of the variance. Therefore, first principal component of our system successfully retained $98 \%$ of the data and we name it Fed Index.

\section{B. Results}

The Fed Index was then normalized by Z-scores and then plotted against the normalized Z- scores of S\&P 500 index in a period of 2006 to 2011 .

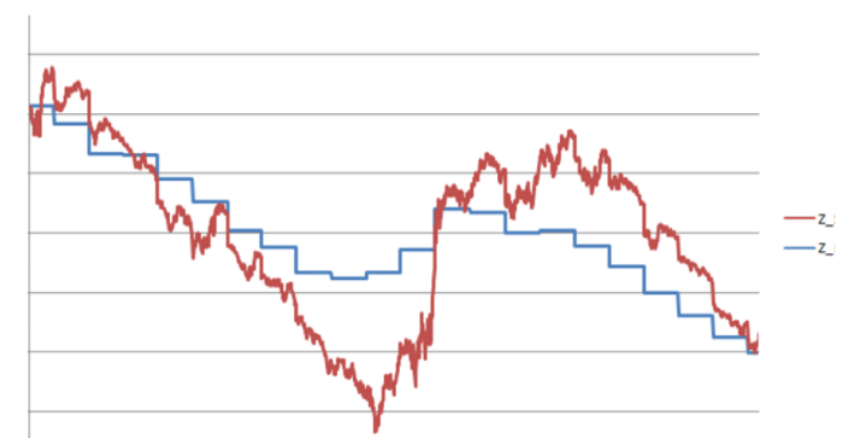

Fig. 1. S\&P with FED INDEX.

The graph (Fig. 1) clearly depicts a close relation of FED INDEX with the S\&P 500 index in the pre and post crisis era. However, the correlation decreases significantly which depicts that the impact of monetary policy in the peak crisis period decreases. None the less, the overall of shape of FED INDEX and the S\&P500 is similar which forms a basis of the ANN Decision support system explained the next section.

\section{ANN DECISION SUPPORT}

In the previous section, we obsereved a good amount of similarity in the principal component of federal economic variables and the market (S\&P 500). Based on that, a decision support system, which will classify the day's federal monetary scenario into SELL, HOLD and BUY day classes is trained.

A new feature is engineered based on the probabilities of the derivatives of the $\mathrm{S} \& \mathrm{P}$ index.

Following is the summary(Fig. 2) of the derivative function of S\&P 500 index, generated by R.

$\begin{array}{rrrrrr}\text { Min. } & \text { 1st Qu. } & \text { Median } & \text { Mean } & \text { 3rd Qu. } & \text { Max. } \\ -90.17000 & -5.22300 & 0.70330 & 0.03089 & 6.43000 & 91.59000\end{array}$

Fig. 2. Summary of S\&P derivative data.

As per the quaterly division, the derivative/change of the day goes beyond or below the two quaterly benchmarks, the day is classified into the three defined categories. An ideal 'Selling day' is when the S\&P 500 is expected to fall more then the 1st qaterly difference (5.22). An ideal buy day is when the market is expected to raise by 3rd Quaterly difference (6.43) and hold day is when the index does'nt fluctuate much.

Making the newly engineered series as our response variable, ANN is trained for categorizing the daily fedral data (combination of the above discribed features). The algorithm used in training of the ANN is conjugate gradient backpropagation.

To give ANN an appropriate depth for the best prediction accuracy, the data is divided randomly into three partsTraining Data, Validation Data and Test Data in 5:3:3.

Ten hidden Neurons are implied to explore complex and non linear relationships of the daily data based on experiments with several.

Fig. 3 shows the basic architecture of the Artificial Neural 
Network used for the classificaiton. Input is processed in the hidden layers for generating desired classes, in our case 3, BUY, HOLD and SELL.

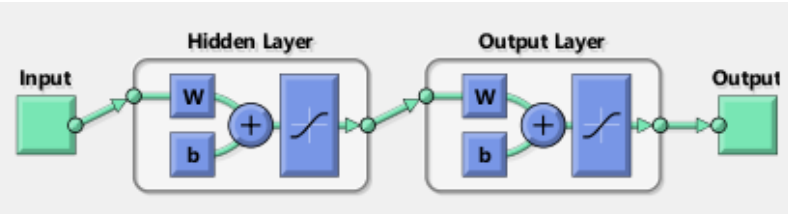

Fig. 3. ANN architecture.

\section{ANN DECISION SUPPORT: RESULTS}

To go to the grassroot level of the crisis by implying machine learing, the dataset is again distributed in three sections. Pre-Crisis period (2006-2007), Crisis Period (Jan 2007-July 2009) and Post-Crisis period (2009-2011).

Let us first start by exploring results of Crisis period.

\section{A. Crisis}

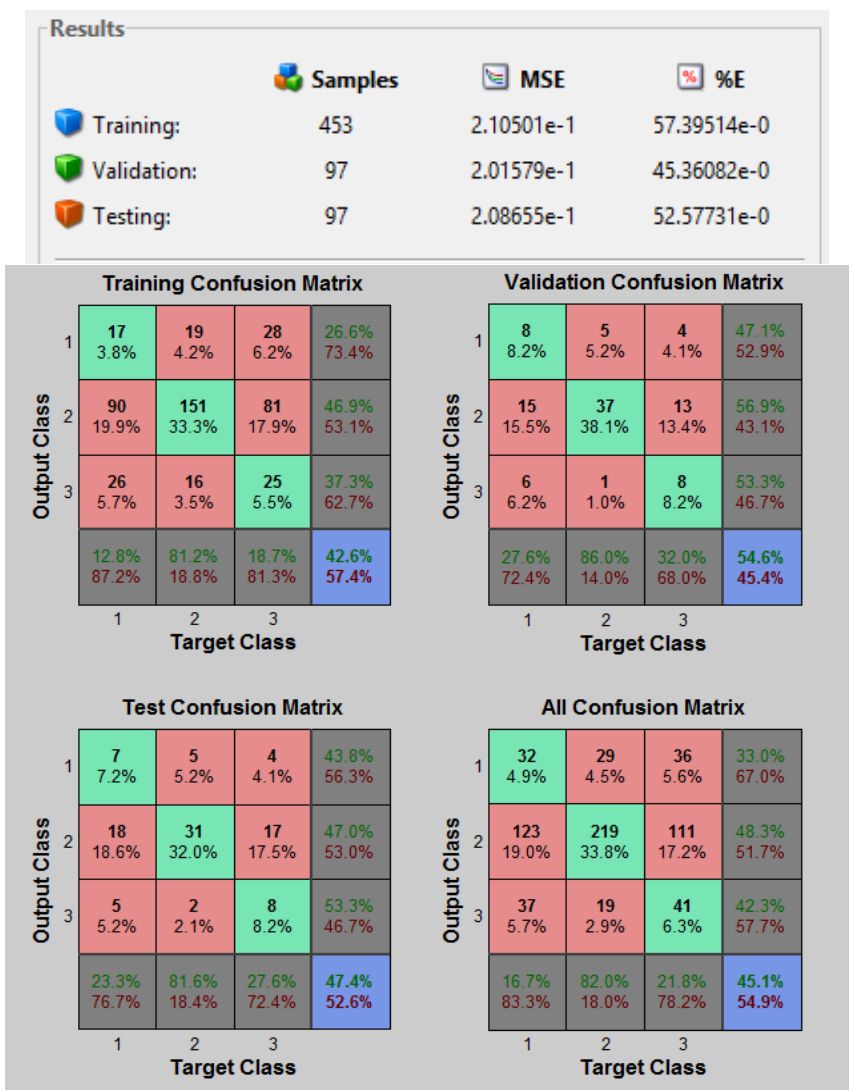

Fig. 4. Results of ANN on crisis period.

The model gives a good accuracy of around 50\%, (refer to Fig. 4) in the major crisis region and it is expected to be lower than the pre and post period and this is alighned with the results of our model.

\section{B. Pre-Crisis Period}

The prediction accuracy of the model substantially increase as compared to the crisis region as the turbulence is low. The model accurately predicts $\sim 70 \%$ (refer to Fig. 5) classification.

\section{Post-Crisis Period}

The post-crisis period also showed improvement in the prediction rate which came out to be $\sim 60 \%$ (Refer to Fig. 6).

$\begin{array}{lccc}\text { Results } & \text { Samples } & \text { 目 MSE } & \text { Q } \\ \text { Training: } & 101 & 1.72925 \mathrm{e}-1 & 33.66336 \mathrm{e}-0 \\ \text { Validation: } & 22 & 1.53546 \mathrm{e}-1 & 27.27272 \mathrm{e}-0 \\ \text { Testing: } & 22 & 1.60093 \mathrm{e}-1 & 31.81818 \mathrm{e}-0\end{array}$
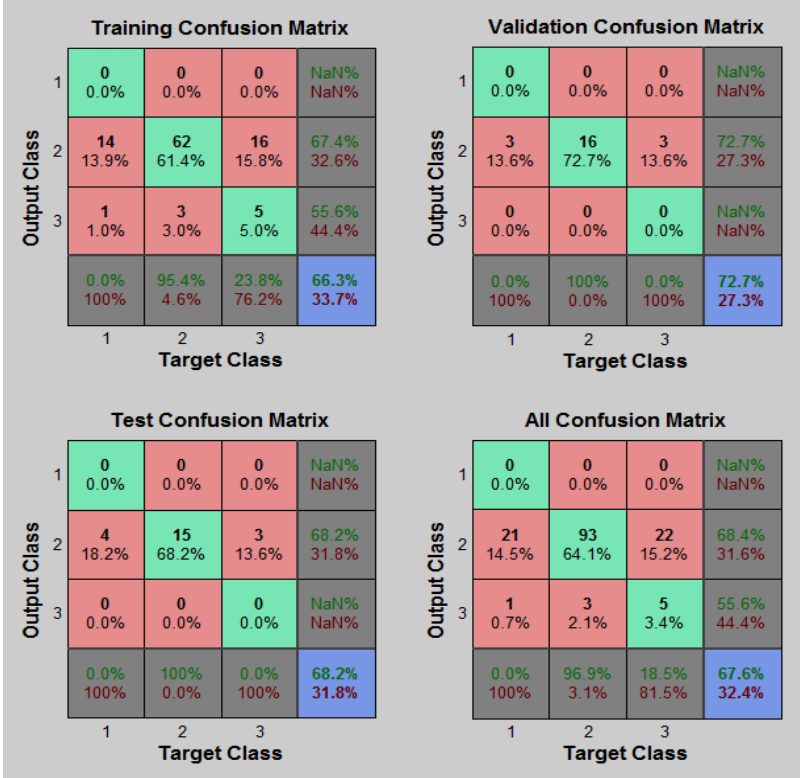

Fig. 5. Results of ANN pre-Crisis period.
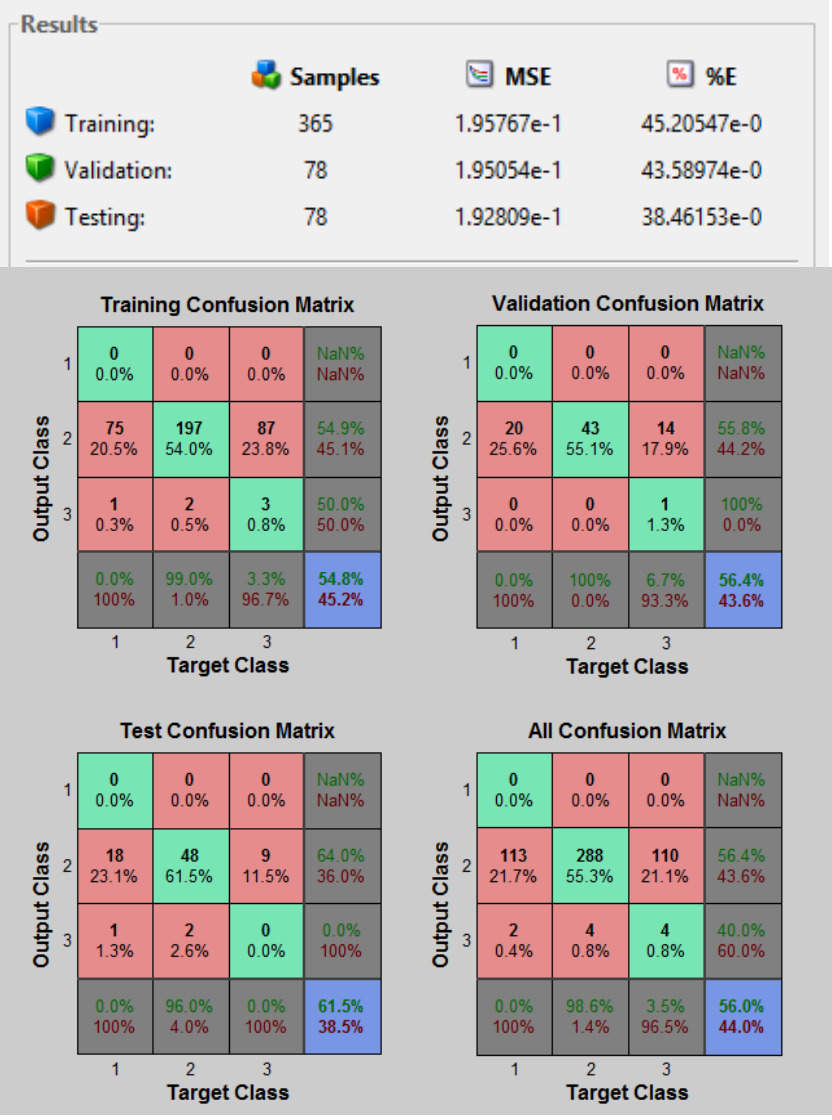

Fig. 6. Results of ANN post-crisis period.

\section{CONCLUSION}

The paper compiles a stepwise progress of the project 
which leads to the development of a new index which summarizes the whole prevailing monetary policy of the US and a decision support system which can help traders and bankers to precisely determine their trading strategies. The paper also examines the impact of the federal policies on the stock market and with the usage of such model, decisions can be simulated by the central bank.

The decision support model gives a good accuracy in non-crisis or recovering phases, which implies the model can be used otherwise as well. The post crisis period in the recovery phase was full of new regulations which created a slight disruption in the model but the model performed quite steadily in the pre crisis period. Post crisis period was full of reforms and new regulations while the pre crisis period was a smooth phase in the history of the financial world. Therefore, the model performed best in the pre-crisis phase and relatively better in the post-crisis phase.

It has been well established that psychological factors play an extremely important part in defining the market in the crisis times (both boom and bust). Therefore, a psycological index, which can be created by sentiment analysis of social media, media and surveys can significantly improve the prediction accuracy of the above-defined algorithm and therefore it forms a prominent future scope for this project.

Non-linear data dimentionality reduction techniques can also be used to summarize the monetary policy into a single elegant number which can reflect the current shape of the market. Slightly more complicated statistical methods can determine the non-linear boundaries in the data which could prove to be better than the linear technique (Principal Component Analysis) used in the paper.

\section{REFERENCES}

[1] R. J. Shiller, Speculative Asset Prices, Stockholm University, 2013.

[2] C. Van James and G. C. George, The Random Walk Theory: An Empirical Test, Parker, Horne, 1967.

[3] S. Basu, Investment Performance of Common Stocks in Relation to Their Price-Earnings Ratio: A Test of the Efficient Market Hypothesis, 1977.

[4] T. D. Sanger, Optimal Unsupervised Learning in a Single-Layer Linear, Pergamon Press Plc, 1989.

[5] H.-N. Robert, Theory of the Backpropagation Neural Network.

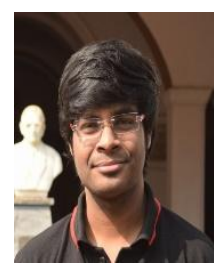

Vyom Shrivastava was born in Indore, India. He is a 4th year undergraduate student of the Department of Mechanical Engineering, Indian Institute of Technology, Kharagpur, India. He is interested in exploring the synergy of technology and finance.

$\mathrm{He}$ had interned at University of Cambridge, Australian National University, IIM Ahmedabad and Trexquant Investment LP (Hedge Fund) exploring the interaction of technology with finance. 